\title{
EXTREME RAYS OF CERTAIN CONES OF HERMITIAN FORMS
}

\author{
DRAGOMIR Ž. DJOKOVIĆ
}

\begin{abstract}
Let $\mathcal{X}$ be the real vector space of hermitian forms on a finite-dimensional complex vector space $V$. For $f \in \mathcal{K}$ we denote by $\mathcal{I C}(f)$ the closed convex cone in $\mathscr{K}$ consisting of forms $g$ such that $g(x, x)>0$ for all $x$ satisfying $f(x, x)>0$. Unless $f<0$ and $f \neq 0$, the cone $\mathscr{T}(f)$ contains no nonzero subspaces of $\mathcal{K}$. Assuming that this is the case, we determine the extreme rays of $\mathcal{H}(f)$. The same problem is solved for real and quaternionic spaces.
\end{abstract}

0. Introduction. Let $V$ be a (right) vector space over $\mathbf{F}$, where $\mathbf{F}$ is one of the following: the real field $\mathbf{R}$, the complex field $\mathbf{C}$, or the real quaternions $\mathbf{H}$. We shall denote by $\mathcal{H}=\mathcal{H}_{V}$ the real vector space of hermitian forms $f: V \times V \rightarrow F$ (if $\mathbf{F}=\mathbf{R}$ then $f$ is in fact a symmetric bilinear form). We shall write $f(x)$ instead of $f(x, x)$ when $f \in \mathcal{H}$, and recall that $f(x) \in \mathbf{R}$ for all $x \in V$. We denote by $V_{f}$ (resp. $V_{f}^{+}, V_{f}^{-}$) the set of vectors $x \in V$ satisfying $f(x)=0$ (resp. $f(x)>0, f(x)<0$ ).

For $f \in \mathcal{K}$ we say that $f$ is positive semidefinite $(f \geq 0)$ if $V_{f}^{+}=V$; it is negative semidefinite $(f \leqslant 0)$ if $-f \geqslant 0$; it is positive definite $(f>0)$ if $f>0$ and $V_{f}=\{0\}$; it is negative definite $(f<0)$ if $-f>0$; and it is indefinite if $V_{f}^{+} \neq V \neq V_{f}^{-}$. If $f$, $g \in \mathcal{H}$ then we also write $f \geqslant g$ (resp. $f>g$ ) to mean that $f-g>0$ (resp. $f-g>0$ ).

We are mainly interested in the finite-dimensional case. Let $\operatorname{dim}_{F} V=n<\infty$. For $f \in \mathcal{H}$ we define its signature by

$$
\operatorname{sign}(f)=\left(n_{-}, n_{0}, n_{+}\right)
$$

where $n_{-}$(resp. $n_{+}$) is the maximum dimension of a negative definite (resp. positive definite) subspace of $V$ and $n_{-}+n_{0}+n_{+}=n$. Thus $n_{-}+n_{+}=r$ is the rank of $f$, and $n_{0}$ is the dimension of its radical $\operatorname{Rad} f$. Recall that $\operatorname{Rad} f=\{a \in V \mid f(a, x)=$ $0, \forall x \in V\}$.

For a fixed $f \in \mathcal{H}$ let $\mathcal{H}(f)$ denote the set of all forms $g \in \mathcal{H}$ satisfying the condition $V_{f}^{+} \subset V_{g}^{+}$. It is clear that $\mathcal{H}(f)$ is a closed convex cone in $\mathscr{H}$ (with vertex at the origin). Since all positive definite forms belong to $\mathcal{H}(f)$, this cone has nonempty interior. It is easy to verify that the condition

$$
\mathcal{H}(f) \cap(-\mathcal{H}(f))=\{0\}
$$

Received by the editors July 24, 1980.

1980 Mathematics Subject Classification. Primary 15A63, 46D05; Secondary 15A33, 15 A47.

Key words and phrases. Hermitian form, positive definite, signature, radical, real quaternions, convex cone, extreme ray.

'This work was supported in part by NSERC Grant A-5285.

() 1981 American Mathematical Society 0002-9939/81/0000-0454/\$02.25 
is satisfied iff either $f \$ 0$ or $f=0$. We shall assume throughout that (1) holds, but see the remark at the end for the exceptional case.

A ray is a set $[g]=\{\lambda g \mid \lambda \geqslant 0\}$ where $g \in \mathcal{H}$ and $g \neq 0$. A ray $[g] \subset \mathcal{H C}(f)$ is an extreme ray of $\mathcal{H}(f)$ if $g=g^{\prime}+g^{\prime \prime}$ and $g^{\prime}, g^{\prime \prime} \in \mathcal{H}(f)$ imply that $g^{\prime}, g^{\prime \prime} \in[g]$. The properties of $\mathcal{H}(f)$ mentioned above imply that $\mathscr{H}(f)$ is the convex hull of the set of its extreme rays. The man objective of this paper is to determine the extreme rays of $\mathcal{H}(f)$.

Let $\mathcal{P}$ be the set of all forms $g \in \mathcal{H}$ such that $g>0$. By $\mathscr{P}_{1}$ we denote the subset of $\mathscr{P}$ consisting of forms of rank one. If $a \in V$ then we define a form $f_{a} \in \mathscr{C}$ by

$$
f_{a}(x, y)=f(x, a) f(a, y) .
$$

Clearly $\operatorname{rank}\left(f_{a}\right)<1$, and $f_{a}=0$ iff $a \in \operatorname{Rad} f$. It is also clear that $f_{a} \in \mathscr{P}$.

We can now state our main result. If $f>0$ then $\mathcal{H}(f)=\mathscr{P}$ and it is well known that the extreme rays of $\mathscr{P}$ are $[g]$ for $g \in \mathscr{P}_{1}$. If $n_{-}>1$ and $n_{+}>2$ then the extreme rays of $\mathcal{H}(f)$ are the extreme rays of $\mathcal{P}$ and the ray $[f]$. Finally if $n_{-}>1$ and $n_{+}=1$ then the answer is the same as in the previous case except that the rays $\left[f_{a}\right]$ for $f(a)>0$ are not extreme.

The main tool in our proof is a theorem of Krein and Šmul'jan concerning a pair of hermitian forms. In the first section we give a brief account of that theorem as well as a few related theorems. Perhaps Theorem 4 in that section is new. In the second section we state and prove our main result.

1. Comparison of hermitian forms. The following basic result is due to $R$. Kühne [2] and M. G. Krein and Ju. L. Šmul'jan [1].

TheOrem 1. If $f \in \mathcal{K}_{V}$ is indefinite and $g \in \mathcal{H}_{\text {satisfies }} V_{f} \subset V_{g}^{+}$then

$$
g(x) / f(x)>g(y) / f(y)
$$

holds if $f(x)>0$ and $f(y)<0$.

The proof given in [1] assumes that $\mathbf{F}=\mathbf{C}$. The case $\mathbf{F}=\mathbf{H}$ reduces to the case $\mathbf{F}=\mathbf{C}$ by restricting scalars. The observation that this result is valid also in the real case is apparently due to J. Bognar; see the addendum to [3]. We give the proof in the real case by modifying the corresponding proof in [1].

Assume that (2) is violated for some $x, y$. We may assume that $f(x)=1$ and $f(y)=-1$, and so by hypothesis $g(x)+g(y)<0$. Let $z=x+t y, t \in \mathbf{R}$. We have $f(z)=1+2 t f(x, y)-t^{2}$. This polynomial has real roots $t_{1}, t_{2}$ and they satify $t_{1} t_{2}=-1$. We also have

$$
g(z)=g(x)+t^{2} g(y)+2 \operatorname{tg}(x, y)<\left(t^{2}-1\right) g(y)+2 \operatorname{tg}(x, y) .
$$

Since $\left(t_{1}^{2}-1\right) g(y)+2 t_{1} g(x, y)=-t_{1}^{2}\left[\left(t_{2}^{2}-1\right) g(y)+2 t_{2} g(x, y)\right]$, we can choose $t=t_{1}$ or $t=t_{2}$ so that $\left(t^{2}-1\right) g(y)+2 \operatorname{tg}(x, y) \leqslant 0$. Then we have $f(z)=0$ and $g(z)<0$, which is a contradiction.

The following two theorems are easy consequences of Theorem 1 . When $\mathbf{F}=\mathbf{C}$ they were proved in [1] and in other cases the proofs are similar. 
TheOREM 2. Let $f, g \in \mathcal{H}_{V}$ and assume that $f$ is indefinite and $V_{f} \subset V_{g}^{+}$. Then $\lambda:=\inf _{f(x)=1} g(x)>-\infty$ and $g>\lambda f$. If moreover $V_{f}^{+} \subset V_{g}^{+}$then $\lambda>0$.

TheOREM 3. Let $f, g \in \mathcal{K}_{V}$ and assume that $f$ is indefinite and $V_{f} \subset V_{g}$. Then there exists $\sigma \in \mathbf{R}$ such that $g=\sigma f$.

The following theorem we were not able to locate in the literature. (The referee points out that it is an easy consequence of part II of [5, Main Theorem].)

TheOREM 4. Let $V$ be a finite-dimensional $\mathbf{F}$-vector space $(\mathbf{F}=\mathbf{R}, \mathbf{C}$, or $\mathbf{H})$ and $f, g$ hermitian forms on $V$. If $V_{f}^{+} \subset V_{g}^{+}$and $V_{f}^{+} \cap V_{g}=\{0\}$ then there exists a constant $\lambda>0$ such that $g>\lambda f$.

Proof. By Finseler's theorem (see [5, Main Theorem]) $f$ and $g$ are simultaneously diagonalizable, i.e., there exists a basis $e_{1}, \ldots, e_{n}$ of $V$ such that $f\left(e_{r}, e_{s}\right)=$ $g\left(e_{r}, e_{s}\right)=0$ for $r \neq s$. Let $f\left(e_{r}\right)=\alpha_{r}, g\left(e_{r}\right)=\beta_{r}$. We may assume that $\alpha_{r}>0$ for $1<r \leqslant k, \alpha_{r}=0$ for $k<r \leqslant m$, and $\alpha_{r}<0$ for $m<r<n$. Since $e_{r} \in V_{f}^{+}$for $r<m$, we have $\beta_{r}>0$ for $r<m$. Now let $1<r<k, m<s<n$, and

$$
x=\sqrt{-\alpha_{s}} e_{r}+\sqrt{\alpha_{r}} e_{s} .
$$

We have $f(x)=0$, and so $g(x)=\alpha_{r} \beta_{s}-\alpha_{s} \beta_{r}>0$. Since $\alpha_{r}>0$ and $\alpha_{s}<0$, we obtain that $\beta_{s} / \alpha_{s}<\beta_{r} / \alpha_{r}$. Consequently there exists $\lambda>0$ such that $\beta_{s} / \alpha_{s}<\lambda<$ $\beta_{r} / \alpha_{r}(1<r \leqslant k, m<s \leqslant n)$. For this $\lambda$ we have $\beta_{r}>\lambda \alpha_{r}(1<r<n)$ and so $g>\lambda f$.

2. Main result. Our main result is the following theorem.

TheOReM 5. Let $f \in \mathcal{K}_{V}, \operatorname{dim}_{F} V=n<\infty, \operatorname{sign}(f)=\left(n_{-}, n_{0}, n_{+}\right)$and $n_{+}>1$ or $f=0$.

If $f \geq 0$ then $\mathcal{H}(f)=\mathscr{P}$ and its extreme rays are $[g]$ for $g \in \mathscr{P}_{1}$.

If $n_{-} \geqslant 1$ and $n_{+} \geqslant 2$ then the extreme rays of $\mathcal{H}(f)$ are those of $\mathscr{P}$ and the ray $[f]$.

If $n_{-} \geqslant 1$ and $n_{+}=1$ then the extreme rays of $\mathcal{C}(f)$ are $[f]$ and those extreme rays of $\mathcal{P}$ which are not of the form $\left[f_{a}\right]$ where $f(a)=1$.

Let us first state two well-known lemmas.

LEMMA 1. If $f \in \mathcal{H}_{V}$ and $f>0$ ( $V$ may be infinite-dimensional) then $f(a)=0$ implies that $a \in \operatorname{Rad} f$.

LEMMA 2. If $\operatorname{dim}_{\mathrm{F}} V=n<\infty$ then the extreme rays of the cone $\mathcal{P}$ are $[f]$, $f \in \mathscr{P}_{1}$.

Proof of Theorem 5. If $f \geqslant 0$ then the claim follows from Lemma 2. Thus we shall assume from now on that $n_{-} \geqslant 1$.

Let $[g]$ be an extreme ray of $\mathcal{H}(f)$. Since $g \in \mathcal{H}(f)$, i.e., $V_{f}^{+} \subset V_{g}^{+}$, it follows from Theorem 2 that $g \geqslant \lambda f$ for some $\lambda \geqslant 0$. Hence $g=\lambda f+h$ where $h \in \mathcal{P}$. Since $[g]$ is an extreme ray of $\mathcal{H}(f)$ and $f \notin \mathscr{P}$, we must have $g=\lambda f$ (and then $\lambda>0)$ or $g=h$. Consequently, either $[g]=[f]$ or $g \in \mathscr{P}_{1}$. 
Now assume that $g=f_{a}$ for some $a \in V$ with $f(a)=1$. We claim that $n_{+}>2$. Assume that $n_{+}=1$. There exists a basis $a_{1}, \ldots, a_{n}$ of $V$ such that $a_{1}=a$, $f\left(a_{r}, a_{s}\right)=0$ for $r \neq s$, and $f\left(a_{r}\right)=0$ or -1 for $r>2$. Then it is easy to verify that $f=f_{a}-f_{2}-f_{3}-\cdots-f_{n}$ where we write $f_{r}$ instead of $f_{a}$ for $r>2$. This contradicts the extremality of $[g]$ since $f \notin \mathscr{P}$ and $f_{r} \neq 0$ for at least one $r>2$.

Conversely, we shall now show that the rays listed in the theorem are indeed extreme rays of $\mathcal{H}(f)$. It is clear that $[f]$ is extreme because $f \notin \mathscr{P}$. Now let $n_{+}>2$ and $g \in \mathscr{P}_{1}$. Since $\operatorname{Rad} g$ is a hyperplane of $V$ and $n_{+}>2$, there exists $x \in V$ such that $g(x)=0$ and $f(x)>0$. By [4, Corollary 18.5.2, p. 167] $\mathcal{H}(f)$ is the convex hull of the union of its extreme rays. Assume that

$$
g=\lambda f+\lambda_{1} h_{1}+\cdots+\lambda_{m} h_{m}
$$

where $\lambda \geqslant 0, \lambda_{r}>0, h_{r} \in \mathscr{P}_{1}$, and $\left[h_{r}\right] \neq[g]$ for $1<r<m$. Since $h_{r}(x) \geqslant 0$, $f(x)>0$, and $g(x)=0$, it follows that in fact $\lambda=0$. The resulting equality $g=\lambda_{1} h_{1}+\cdots+\lambda_{m} h_{m}$ contradicts Lemma 2 . Hence $[g]$ must be an extreme ray of $\mathscr{H}(f)$.

Finally, let $n_{+}=1, g \in \mathscr{P}_{1}$ and assume that $[g]$ is not of the form [ $f_{a}$ ] where $f(a)=1$. We shall refer to rays of this form as admissible. We have to show that $[g]$ is an extreme ray of $\mathcal{H}(f)$. If there exists a vector $x \in \operatorname{Rad} g$ such that $f(x)>0$ then we can proceed as in the case $n_{+}>2$. Thus we may assume that $\operatorname{Rad} g$ is negative semidefinite with respect to the form $f$. Since $n_{+}>1$, this implies that $\operatorname{Rad} g \supset \operatorname{Rad} f$. Consequently there exists a vector $a \in V$ such that $\operatorname{Rad} g=\{x \in$ $V \mid f(a, x)=0\}$. By normalizing $a$, we may assume that $g=f_{a}$. Since $[g]$ is admissible, we have $f(a) \leqslant 0$. Since $\operatorname{Rad} g$ is negative semidefinite with respect to $f$, and $n_{+} \geqslant 1$, we have in fact $f(a)=0$. By the definition of $a$, we infer that $a \in \operatorname{Rad} g$. Assume again that (3) holds under the same conditions as in the previous case. Since $g(a)=f(a)=0$ and $h_{r}(a)>0$ for all $r$, it follows from (3) that $h_{r}(a)=0$ for all $r$. By Lemma 1 we have $a \in \operatorname{Rad} h_{r}$ for all $r$. Since also $a \in \operatorname{Rad} g$ but $a \notin \operatorname{Rad} f$, the equation (3) implies that $\lambda=0$. Now we reach a contradiction as in the previous case. Hence (3) is impossible, i.e., $[g]$ is an extreme ray of $\mathcal{H}(f)$.

This completes the proof of the theorem.

REMARK. In the exceptional case when $f \leqslant 0$ and $f \neq 0$ the cone $\mathcal{H}(f)$ contains a nonzero vector subspace. In fact $\mathcal{H}_{0}(f)=\mathcal{H}(f) \cap(-\mathcal{H}(f))$ is the largest vector subspace contained in $\mathcal{H}(f)$. If $U=\operatorname{Rad} f$ then $\mathcal{H}(f)$ depends only on $U$; it consists of all forms $g \in \mathcal{H}$ for which $U$ is positive semidefinite. The subspace $\mathcal{H}_{0}(f)$ consists of all forms $g \in \mathcal{H}$ for which $U$ is totally isotropic. Fix a direct decomposition $V=U \oplus W$. Let $\mathcal{P}(W)$ be the cone of all forms $g \in \mathcal{P}$ such that $W \subset \operatorname{Rad} g$. This cone can be identified in the obvious way with the cone of positive semidefinite forms on $U$. The structure of the cone $\mathcal{H}(f)$ is now described by noting that $\mathcal{H}(f)=\mathcal{H}_{0}(f)+\mathscr{P}(W)$ and $\mathcal{H}_{0}(f) \cap \mathscr{P}(W)=\{0\}$. Of course the cone $\mathcal{H}(f)$ itself does not have any extremal rays since $\mathcal{H}_{0}(f) \neq\{0\}$.

Finally I wish to thank B.-S. Tam for instigating my interest in the theory of cones and for correcting a mistake in the original proof of the real case of Theorem 1. 


\section{REFERENCES}

1. M. G. Krein and Ju. L. ŚSmul'jan, Plus-operators in a space with indefinite metric, Mat. Issled. 1 (1) (1966), 131-161 = Amer. Math. Soc. Transl. (2) 85 (1969), 93-113.

2. R. Kühne, Über eine Klasse J-selbstadjungierter Operatoren, Math. Ann. 154 (1964), 56-69.

3. R. Loewy and H. Schneider, Positive operators on the n-dimensional ice cream cone, J. Math. Anal. Appl. 49 (1975), 375-392.

4. R. T. Rockafellar, Convex analysis, Princeton Univ. Press, Princeton, N. J., 1970.

5. F. Uhlig, A recurring theorem about pairs of quadratic forms and extensions: $A$ survey, Linear Algebra Appl. 25 (1979), 219-237.

Department of Pure Mathematics, University of Waterloo, Waterloo, Ontario, N2L 3G1, Canada 